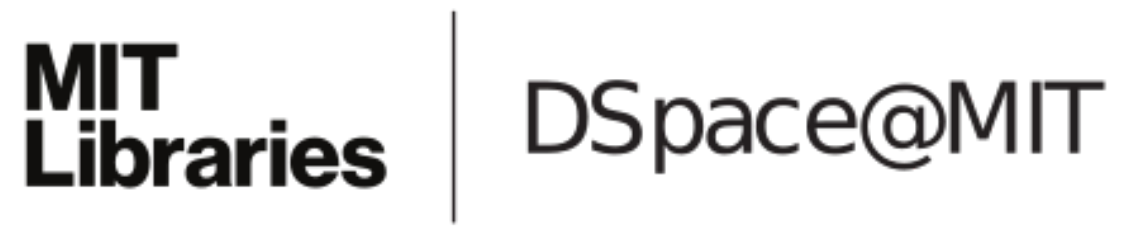

\author{
MIT Open Access Articles
}

Design of a Growing Robot Inspired by Plant Growth

The MIT Faculty has made this article openly available. Please share how this access benefits you. Your story matters.

Citation: Yan, Tongxi et al. "Design of a Growing Robot Inspired by Plant Growth." IEEE International Conference on Intelligent Robots and Systems, November 2019, Macau, China, Institute of Electrical and Electronics Engineers, January 2020. (C) 2019 IEEE

As Published: http://dx.doi.org/10.1109/iros40897.2019.8968200

Publisher: Institute of Electrical and Electronics Engineers (IEEE)

Persistent URL: https://hdl.handle.net/1721.1/127834

Version: Author's final manuscript: final author's manuscript post peer review, without publisher's formatting or copy editing

Terms of use: Creative Commons Attribution-Noncommercial-Share Alike 


\title{
Design of a Growing Robot Inspired by Plant Growth*
}

\author{
Tongxi Yan ${ }^{1}$, Seiichi Teshigawara ${ }^{1}$, and H. Harry Asada, Member, IEEE ${ }^{1}$
}

\begin{abstract}
A novel design concept of expandable robotic arm inspired by plant growth is presented. The robot can construct its own structure by converting a type of fluidized material into a rigid structure at its growing point. The robot can extend its structure in multiple directions, and move through a winding space to reach a point, which is otherwise difficult to access. The robot with the rigid structure can also bear a significant load, has a plate to attach an end-effector, and can transport an object. The robot satisfies three key functional requirements that are characteristic to plant growth. First, the robot is capable of transporting structural materials to its growing point. Second, the robot is capable of transforming the material into a rigid structure. Third, it is capable of steering its growing point so that it is expanded in a desired direction. A proof-ofconcept prototype is then presented that consists of a special sprocket chain that can be switched between flexible/fluidized and rigid states, a winch that can pull/transport the chain, and a steering system to direct the growing direction. Unlike plants, this growing robot can retract its extended body, and can extend in a different direction. The prototype demonstrates that it meets all the functional requirements, and that it can make sharp turns and move through obstacles.
\end{abstract}

Keywords: Industrial Robots, Mechanism Design

\section{INTRODUCTION}

In inspection, maintenance, and assembly of complex machines and systems, robots often have to reach objects through a narrow, winding space. In logistics automation, robots also need to pick up goods at the back of a shelf in a warehouse. This type of tasks, which is referred to as the Last One-Foot Problem, is posing a challenge to robot design and control [1]. Traditional robots consisting of a series of servoed joints or a parallel linkage structure are unable to reach such destinations.

In the robotics community, a number of innovative robots having unique body shapes and characteristics have been investigated. These include soft robots, mobile robots, and motion planning techniques utilizing shrinking and growing geometries that can be applied to the last one-foot problem [1]-[6]. Of particular interest is the soft mobile robot by the group at Stanford University [2]. It resembles a plant that can extend its body through a narrow channel. Unfortunately, however, these robots are often unable to bear a large load. Soft robots, in particular, cannot position their endeffectors precisely at a desired point in space. They do not even possess an appropriate base to mount an end-effector. Furthermore, mobile robots in general are difficult to use in a practical setting, since they are unable, or find it difficult,

*This work was supported by NSK Ltd.

${ }^{1}$ The authors are with the d'Arbeloff Laboratory for Information Systems and Technology in the Department of Mechanical Engineering, Massachusetts Institute of Technology, Cambridge, MA 02139, USA. Email: \{tyan, seiichi, asada\}@mit.edu to reach the entrance of such a cluttered area. Our view is that some type of end-effector or a small robot attached to the tip of a robot will be a feasible, cost-effective solution.

To build a robust robot structure for the above application, studies on the mechanism of how a plant grows are conducted. A plant consists of two main systems called shoot and root systems (see Fig. 1). Nutrients and water from the soil are absorbed by roots and are delivered to the shoot system through the stem. Combined with sunlight, these materials are transformed into the body of the plant. The plant repeats this process to grow more leaves and become taller.

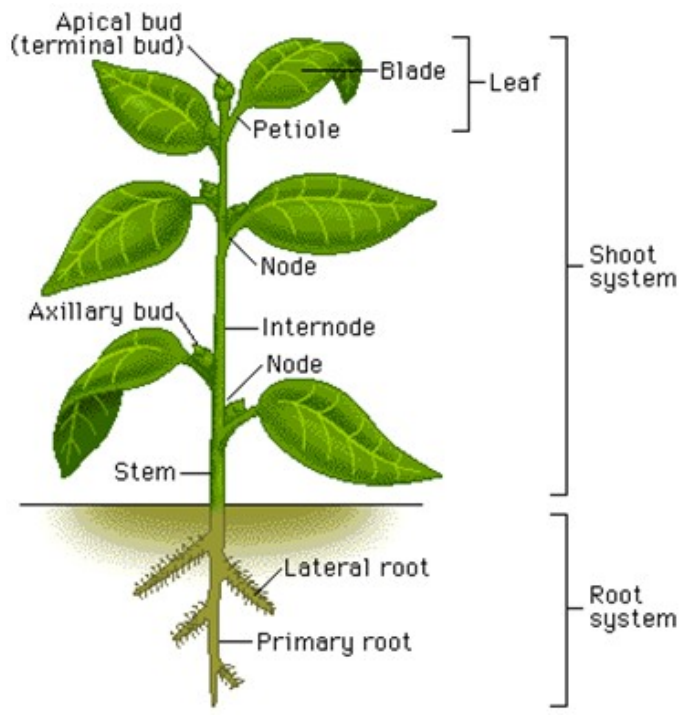

Fig. 1. Structure of a plant, consisting of the root system that delivers materials to the shoot system to grow [7]

There are many studies to support the idea of applying plant growth to robotics. Researchers have developed robots that are inspired by the ability of plant's root to penetrate soil, or granular media [8], [9]. Other robots include tendondriven robots with the ability to bend their bodies, robots inspired by kinematics of vines, and growing achieved by fused deposition modeling [10]-[13]. While these robots are designed to behave like plants in a mechanical way, another approach of incorporating properties of plant growth to a robot without any motion is also studied [14]. However, they do not have good load-bearing capacity, especially at the tip for mounting an end-effector. Therefore, above works not only validate the feasibility of a plant-inspired growing robot, but show what needs to be improved in this field.

The current work on a growing robot is a potential solution 
to the class of the last one-foot problem. It is fully functional on its own, extending from a base and navigate through the environment that human have little to no access to. It can be also mounted on an industrial robot and extends its endpoint into a cluttered space. In either case, the robot can be fully autonomous with proper motion planning algorithms or manually operated, but this is beyond the scope of this paper. However, the basic design concept to be detailed below can go beyond the original mission of the project. The plant-inspired growing robot can construct an arbitrary rigid structure by converting a flexible structural material into a rigid structure. Traditionally, structure is mostly predetermined by design and its configuration is changed by means of active control of servoed joints. In contrast, the new plant-inspired growing robot does not possess fixed link lengths and a fixed kinematic structure. Rather, the structure is determined and constructed in real time. Similar to roots and trunks of a plant, the actual shape is determined through interactions with the environment, which are unpredictable.

The following sections will describe the fundamental functionality of the growing point of a plant, followed by a specific mechanical design concept as an embodiment of the growing point functionality. Then, a preliminary proofof-concept prototype will be designed, fabricated, and tested.

\section{Fundamental Functional REQuirements}

The design needs to be started by turning the functions of a plant to engineering functional requirements. First, as stated above, materials are delivered from the root to the plant body. Once materials are delivered, the plant grows larger. This means that the robot needs to expand from the base, which is equivalent to soil for the plant. Second, the plant constructs its body from the materials. On the robot side, the body must stay rigid after expanding from the base. Third, as the plant grows, it can adapt to different geometries for various reasons. It can grow to avoid obstacles or to gain more sunlight. This needs to be the same for a robot. It needs to be able to steer to different directions in order to avoid obstacles. To summarize, three functional requirements are stated and listed below:

1) Transport materials from a base to a growing point

a) This entails that the material is flexible, so that it can conform to an arbitrary shape of the structure that has been constructed and is amenable for transportation.

b) This also entails a mechanism for pushing, pulling, sucking, pumping, etc. of the material from a base station to the growing point.

2) Convert or transform the material to a rigid structure

a) This entails a mechanism that dispense the materials continually or unit by unit at the tip of the growing point.

b) This also entails a type of locking mechanism, so that the dispensed materials or unit can be immobilized. c) Furthermore, this entails that the dispensing mechanism can push its own body forward, leaving the dispensed materials or unit behind.

3) Steer the growing direction

a) The growing point mechanism must have the means to rotate its body, so that each unit or material can be dispensed in a desired direction.

b) Torque must be generated between the segments of the dispensed materials or units that have been immobilized and the head of the growing point mechanism.

These are fundamental functional requirements for plants as well as for growing robots. Note that the above functional requirements are nothing specific to a particular embodiment. It can be realized with a biological means, or an abiological means. Here we pursue an abiological means, that is, an engineered entity, or a robot. The true value of the above argument of fundamental functional requirements is to release us from considering only existing mechanisms and existing biological systems. There may be other ways of realizing the same functionality using different means.

\section{PRototype Design AND FABRICATION}

A schematic diagram of the robot is demonstrated in Fig. 2. The robot contains four critical parts. The base is the ground from where the robot grows. The constructed structure (red ellipses) is the body of the robot that needs to remain rigid. The fluidized materials (green ellipses) are equivalent to the 'nutrients' of plants that are used to construct robot's structure. They are flexible and not actuated. The growing point transforms fluidized materials to constructed structure so that the robot can grow. It is also capable of steering to different directions to move through curved paths.

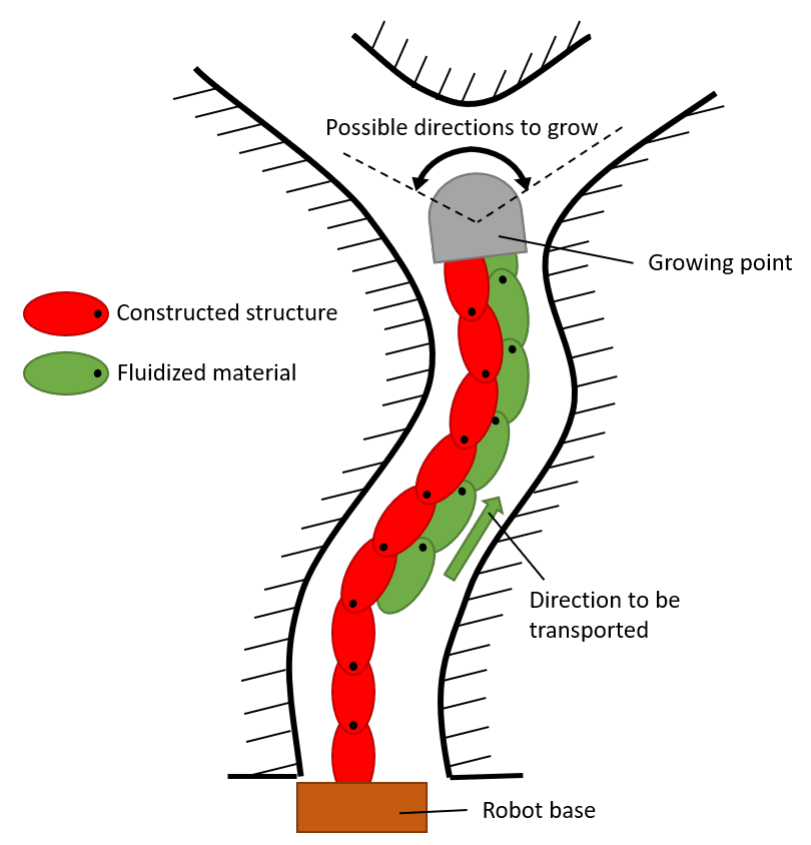

Fig. 2. Schematic of a growing robot with its key components. 
A chain mechanism was chosen as a starting point for the project for its unique potential of being able to stay flexible and rigid. The chain itself is the material of the robot which has to be delivered from the base to a growing point and is used to construct the body. As such, the chain serves as the body of the robot. As the robot expands, the chains that have been deployed to construct the body switch to a rigid state. The rest of the chains remain fluidized until they are used for body construction. An ideal chain would meet the first two functional requirements listed above. The design of a chain block is shown in Fig. 3 below.

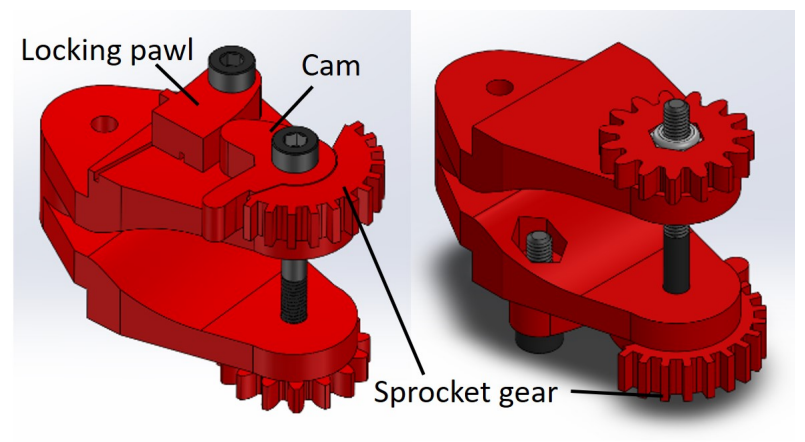

Fig. 3. CAD images of chain block, top (Right) and bottom (left) views.

Both ends are designed so that the chain can be connected in series. In the right figure, there is a gear on the chain for steering the body. This will be explained in detail later. The left figure shows the locking mechanism. Out of many existing locking mechanisms, a device with a latch locking at several different positions [15] was modified and used in the design. A similar joint locking mechanism was applied to the snake robot with a different actuation method [16]. There are two parts associated with the lock, namely the locking pawl and sprocket gear. The locking pawl of one chain is engaged with the teeth of sprocket gear of an adjacent chain. As a result, their rotational motion is constrained, and they become locked together. The lock is activated by the Lshaped pin that acts as a cam. As the cam rotates, its round edge pushes the locking pawl towards the sprocket gear. A torsion spring that provides a restoring force to retract the pawl from the sprocket gear is installed around the rotation axis of the pawl.
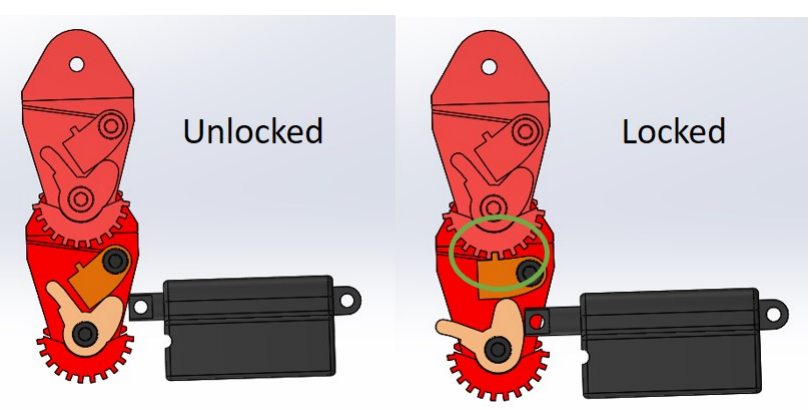

Fig. 4. The locking mechanism and how the linear actuator locks the joint. Green circle shows how two blocks are locked by gear and pawl.
Next, in order to activate the lock, a linear actuator is needed for pushing the cam. It is mounted on the base shown in Fig. 5. The chain moves along the slot which acts as a guide to the chain. The part inside the green circle in Fig.4 is where the linear actuator contacts the cam. As it extrudes out, it pushes the cam that further pushes the locking pawl towards the sprocket gear to activate the lock system. On the base in Fig. 5, there is an unlock pin for transferring rigid materials to flexible state. this is explained in details later.

Other essential parts of the design are the center sprocket for driving the chain, upper housing, and actuators. The assembly of the whole system is shown in Fig. 5 with exploded view to display all parts. Inside the upper housing, the chains, central sprocket, and locking mechanism are enclosed. Two Dynamixel servo motors are mounted on the upper housing. The larger one is to drive the central sprocket and the smaller one steers the robot. The top and bottom housings are bound together by screws. The chain outside the housing is fixed to the ground and it becomes the base from where the robot grows.
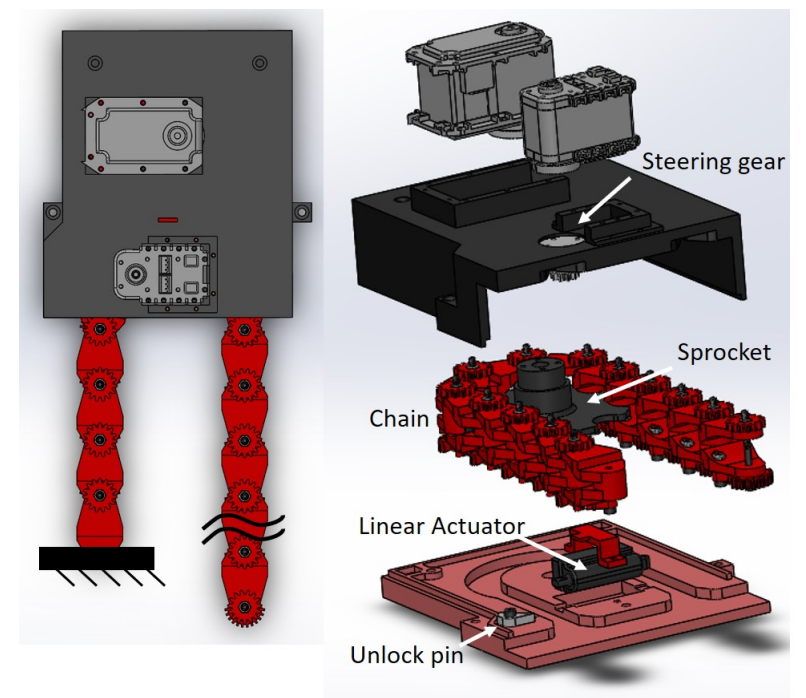

Fig. 5. CAD assembly of the system with an exploded view to show all essential components.

\section{IMPLEMENTATION}

A prototype that can achieve the three functional requirements were assembled based on the presented design. In the sections below, methods to realize desired functional requirements are explained.

\section{A. Transport materials}

Chain blocks are the material for the robot structure and the central sprocket drives the chain to either extend or retract the structure. As shown in Fig. 6, when the sprocket turns, the upper half of the chain moves away from the sprocket and are transformed to part of the robot's body once they are locked. The white gear next to the chain blocks is for steering, which is discussed in detail later. When a chain block passes through the steering gear during either 


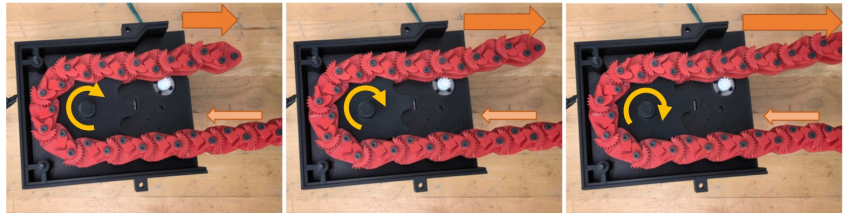

Fig. 6. The middle sprocket rotates (yellow arrow) to feed blocks out (dark orange arrow) from one side and pulls flexible chain from another side (light orange arrow).

extension or retraction, the gear on the chain block meshes with the steering gear. To ensure that gears don't jam during the movement, the steering gear needs to rotate at a speed and direction matching with the speed and direction of the chain's movement. Ideally, the chain is constrained so that it undergoes linear motion when passing through the steering gear, and the relation between the rotational speed of steering gear and the speed of the chain is described as below:

$$
\omega_{\text {gear }}=\frac{2 v_{\text {chain }}}{P D_{\text {gear }}}
$$

where $\omega_{\text {gear }}$ is the rotational speed of the steering gear, $v_{\text {chain }}$ is the linear velocity of the chain, and $P D_{\text {gear }}$ is the pitch diameter of the steering gear. This equation tells how fast the steering gear needs to spin with respect to the moving speed of the chain.

\section{B. Convert materials to rigid structure}

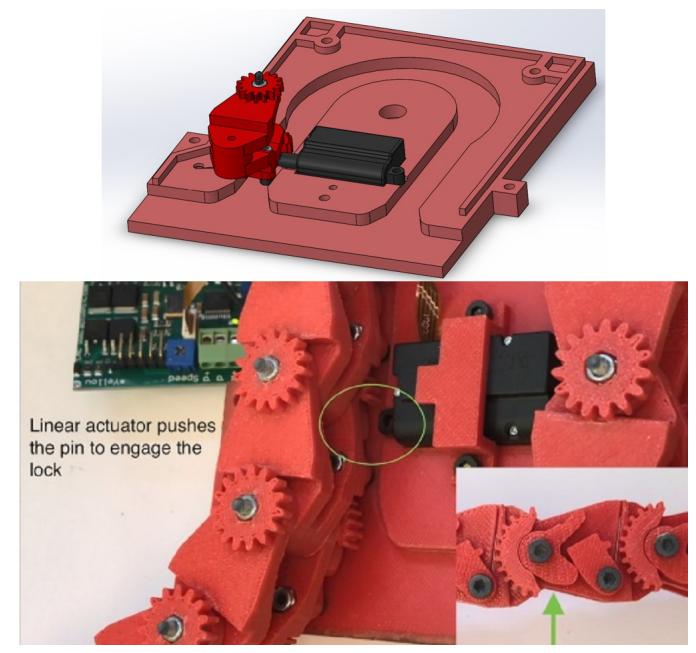

Fig. 7. Method of engaging the lock with CAD image to show where the linear actuator is mounted to the base.

Materials delivered to the growing point of the robot are flexible because they need to fit onto the sprocket. To construct the robot structure, these materials need to be transformed into rigid parts. The lock mechanism on each chain block can constrain the rotation, forming the needed structures. Fig. 7 explains the method of engaging the lock in the system. The linear actuator pushes the cam from the location indicated by the green arrow to lock a block. Once the orientation is determined, its tip is elongated to reach and push the cam.
To unlock the chain, a white pin in Fig. 8 is installed onto the housing base. While unlocking the chain, it also needs to not interfere with locked blocks for constructing the robot body. To achieve this, the pin can rotate in one direction, but not the other, shown by green arrows. When locked blocks are pushed outside the housing, the pin rotates to make space for blocks to pass through and afterwards, it retracts to the original position by a torsion spring embedded on the inside. During the process of retracting the chain, it releases the lock by pushing the cam out of singular position.

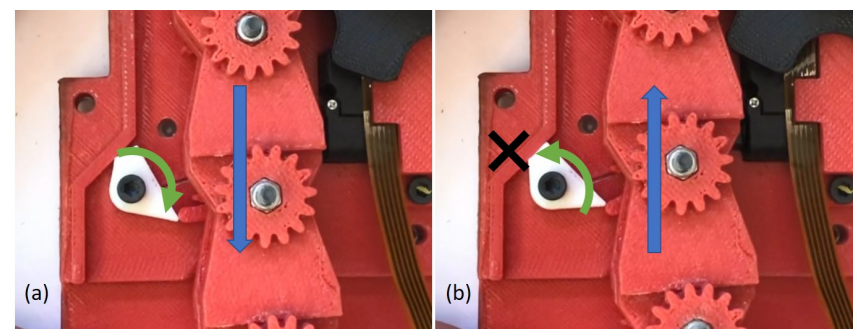

Fig. 8. Unlocking mechanism with blue arrows indicating the direction of chain's motion. (a) As the locked chain passes through unlock pin, the pin rotates clockwise to conform to the motion of chain. (b) When retracting the robot, the pin pushes the cam to release the lock.

\section{Steer the growing direction}

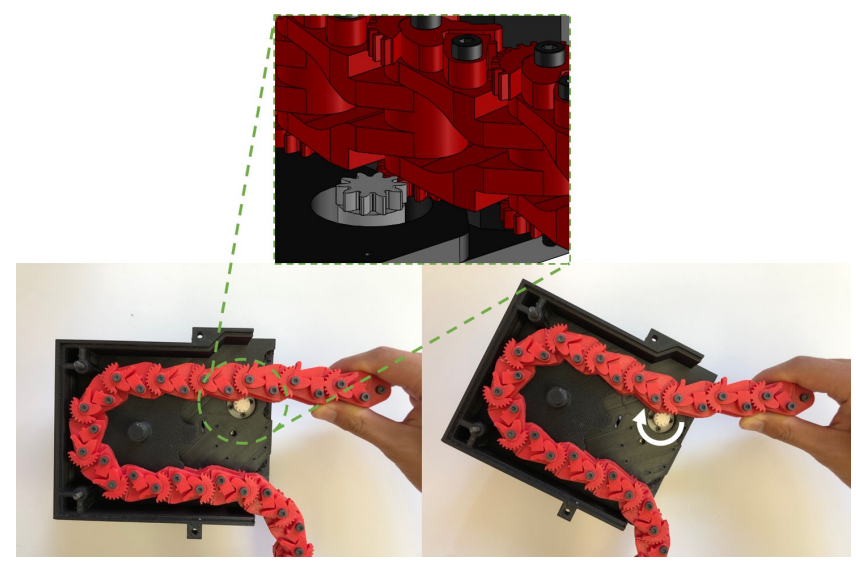

Fig. 9. Steering mechanism. Locked portion of the chain is grounded to the hand. As the white steering gear rotates (white arrow), the whole head tilts to a different direction.

Steering the robot to grow towards the desired direction is the last functional requirement of the design. As briefly mentioned earlier, the steering is achieved by rotating the housing and other components of the robot with respect to the locked structure. The steering gear grounded to the housing meshes with the gear on the locked portion of the chain. As it is driven by a Dynamixel servo motor, it rolls around the gear on a fixed chain. As a result, the whole housing is steered to different directions. Details can be seen in Fig. 9.

\section{EXPERIMENT}

The sequence of operations of this robot is to first feed the chain. Next, the steering gear determines the orientation that 
the robot wants to move to. The chain needs to be locked so that it remains at that orientation, so the linear actuator is driven to lock the chain. Afterwards, the sprocket pushes locked chain outside the housing and builds the body. The same sequence is repeated to expand the robot.

Retracting the robot follows the inverse of the expansion sequence. In order to pull back the chain, the lock needs to be released. Additionally, the chain needs to be repositioned to a straight configuration and this process requires that the chain is unlocked. Therefore, the lock is released first, and then the steering gear rotates to complete the alignment. This is one cycle and is repeated until all body parts are retracted.

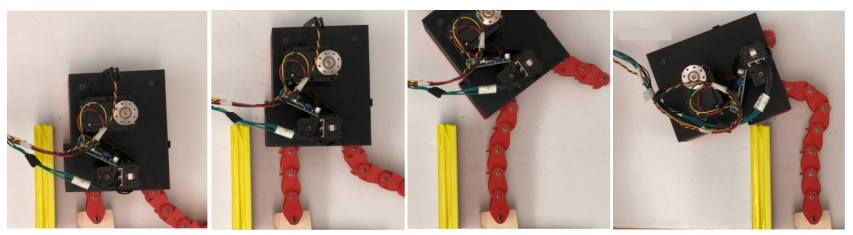

Fig. 10. Demonstration of operating the robot. It first expands straight and then makes a sharp left turn, going around the yellow obstacle.

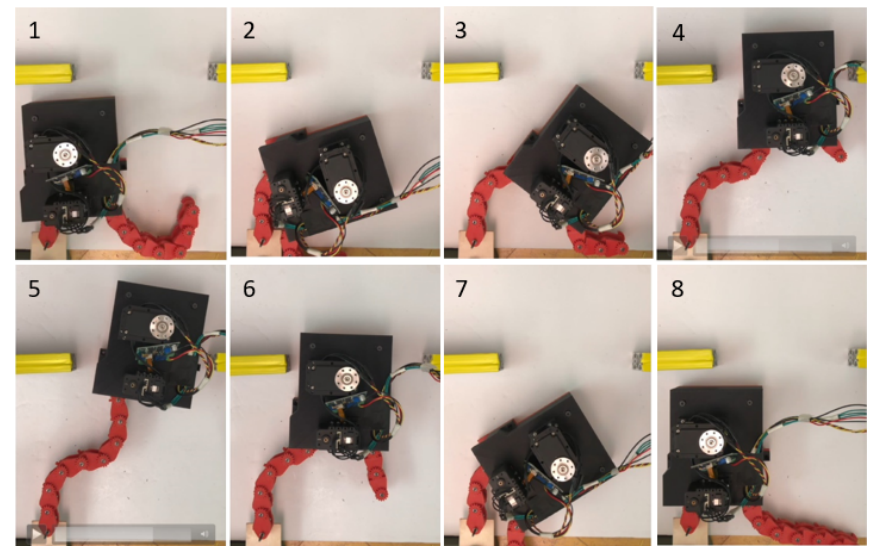

Fig. 11. Demonstration of going through obstacles. The robot is manually operated to go through yellow obstacles and then retract to its original position.

A simple motion of the robot is demonstrated in Fig. 10. The robot first moves straight for several chain blocks. Then, in order to go around the yellow obstacle, the robot makes a left turn. Each chain block can steer up to 60 degrees. The motion demonstrates that the robot can make sharp turns.

Fig. 11 is another demonstration of the robot moving through obstacles. In order to move through the space between two obstacles, the robot needs to first align itself with the space. It makes a right turn and then makes a left turn. After aligning with the space, it moves straight to reach the destination. To show that the robot is capable of retracting to the original position, it pulls its head back to the base by deconstructing the body. Chain blocks are unlocked and retracted one by one during this process and the robot's growing point eventually goes back to the starting position.

\section{DESIGN ITERATIONS}

To reduce backlash and increase locking strength of the current design, the second version of the chain with a new locking mechanism was created. The chain block is assembled from two nearly identical parts, one forming the upper half and the other forming the lower half of a chain block. A bushing is placed between the interface of two chain blocks to reduce friction.
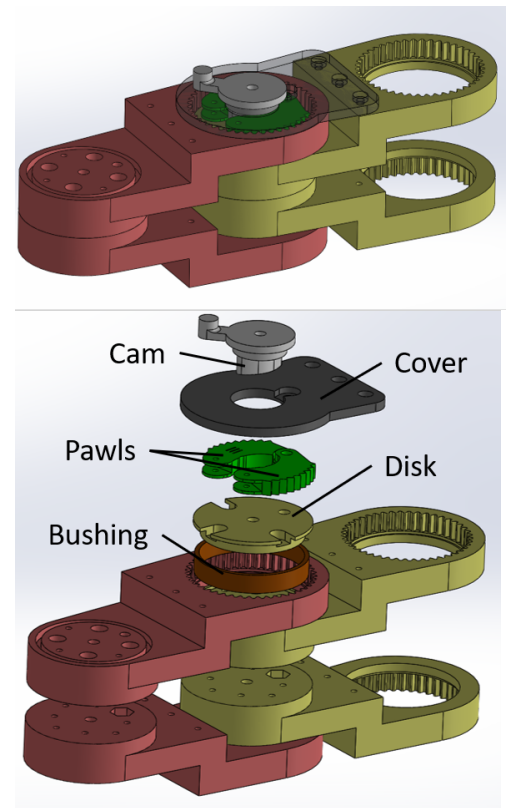

Fig. 12. Second design of chain block and locking mechanism with exploded view. The yellow disk is the mounting plate for pawls and is fixed to the yellow chain block. Note that fasteners are not included and components for the lock are assembled only on top surface. In practice, same components are assembled on bottom surface as well.

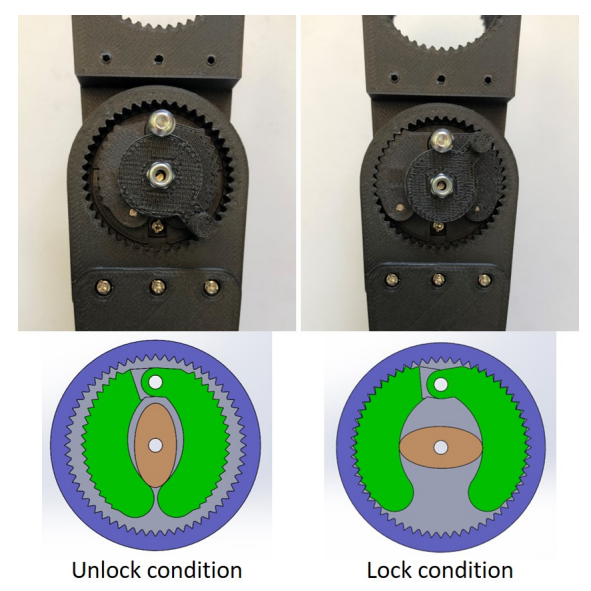

Fig. 13. Locking mechanism of the actual part with CAD images to show the orientation of the cam at each state.

The new locking mechanism consists of two pawls with multiple teeth fixed to a chain block that mesh with an inner gear on the other chain block to engage the lock. Meshing these parts constrains the rotation of two chain blocks about 
each other. The pawls are pushed against the inner gear by a cam sitting between them. Rotating the cam by 90 degrees in either direction can switch between the locked and unlocked states. To separate the pawls from inner gear, a tension spring is attached to the end of two pawls. When the cam no longer pushes the pawls against the inner gear, the springs pulls the pawls to disengage with the inner gear. The cover placed on top of the lock (shown as transparent in the assembled view of Fig. 12) blocks any particles large enough to hinder the locking motion. This lock is on both the top and bottom sides of the chain so that the symmetry reduces twist due to torsion. Having two pairs of lock also enhances the locking strength. A prototype of this design was fabricated and Fig. 13 shows both the locked and unlocked states of the fabricated prototype.

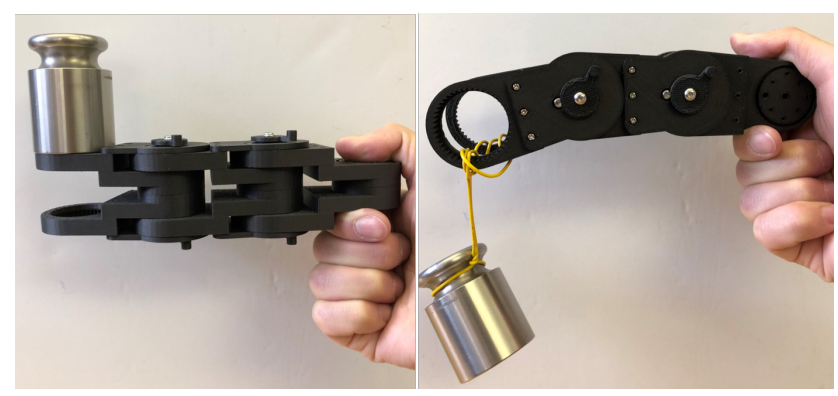

Fig. 14. Demonstration of the load bearing capacity of the second design. The weight carried by the prototype is $500 \mathrm{~g}$

To demonstrate the rigidity of the second design, the prototype consisting of three blocks held a weight of $500 \mathrm{~g}$ in two different orientations. The load was placed at one end, and the the other end was grounded. The first orientation is with the axis of rotation of the chain joint parallel to the load, which in this case is vertical. The load is carried by the structure itself. All joints are locked and the only possible movement is the vertical deflection of the robot. It can be observed that without any support, the prototype can withstand the load. The second orientation is with the axis of rotation is orthogonal to the load. By doing so, the load is carried by locking parts. As seen in Fig. 14, the prototype could resist the load without breaking any components. Further tests will be conducted with more assembled blocks because vertical deflection is proportional to the cube of total length of the structure, $l^{3}$. As the length increases, deflection of the structure becomes larger.

\section{CONCLUSION AND FUture WORK}

The idea and mechanisms behind plant growth can inspire a whole new field of robotic research. This is especially applicable to robots with rigid structures, and innovative designs that possess many advantages compared to conventional robots. Growing structures can adapt to the environment and are less constrained by their surroundings. The first prototype served as a proof of concept and sufficiently proved that plant growth can be incorporated with rigid robotics. It demonstrated the maneuverability of the robot by showing it an move in a range of directions and can retract to its original configuration. The improved design of chain block and locking mechanism can be implemented in a similar way as the first prototype to form a complete robot.

The next step for this project is to complete the whole system using the improved design that achieves all three functional requirements and has better performance than the first prototype in terms of strength, load bearing capacity, and ease of control. A design for constraining fluidized materials is also needed because otherwise, these flexible structures can interfere with surrounding objects and impede the motion of the robot.

\section{REFERENCES}

[1] Shikari, Abbas, and Harry Asada. "Triple Scissor Extender Robot Arm: A Solution to the Last One Foot Problem of Manipulation," IEEE Robotics and Automation Letters 3.4 (2018): 3975-3982.

[2] Hawkes, Elliot W., et al. "A soft robot that navigates its environment through growth," Science Robotics 2.8 (2017): eaan3028.

[3] Putzu, Fabrizio, Taqi Abrar, and Kaspar Althoefer. "Plant-Inspired Soft Pneumatic Eversion Robot," 2018 7th IEEE International Conference on Biomedical Robotics and Biomechatronics (Biorob). IEEE, 2018.

[4] Baginski, Boris. "Local motion planning for manipulators based on shrinking and growing geometry models," Proceedings of IEEE International Conference on Robotics and Automation. Vol. 4. IEEE, 1996.

[5] Stilli, Agostino, Helge A. Wurdemann, and Kaspar Althoefer. "Shrinkable, stiffness-controllable soft manipulator based on a bio-inspired antagonistic actuation principle," 2014 IEEE/RSJ International Conference on Intelligent Robots and Systems. IEEE, 2014.

[6] Saranli, Uluc, Martin Buehler, and Daniel E. Koditschek. RHex: A simple and highly mobile hexapod robot, The International Journal of Robotics Research 20.7 (2001): 616-631.

[7] B. Harmon, "Plant Structure Roots Stems Leaves. Plant Organs Roots Stems Leaves," 2017. [Online]. Available: https://slideplayer.com/slide/10448620/

[8] Del Dottore, Emanuela, et al. "Circumnutations as a penetration strategy in a plant-root-inspired robot." 2016 IEEE International Conference on Robotics and Automation (ICRA). IEEE, 2016.

[9] Sadeghi, Al, et al. "Robotic mechanism for soil penetration inspired by plant root." 2013 IEEE International Conference on Robotics and Automation. IEEE, 2013.

[10] Wooten, Michael, et al. "Exploration and Inspection with VineInspired Continuum Robots." 2018 IEEE International Conference on Robotics and Automation (ICRA). IEEE, 2018.

[11] Wooten, Michael, and Ian Walker. "Vine-inspired continuum tendril robots and circumnutations." Robotics 7.3 (2018): 58.

[12] Truong-Thinh, Nguyen, and Nguyen Ngoc-Phuong. "Design and development of continuum structure for robotic flower." 2011 IEEE International Conference on Robotics and Biomimetics. IEEE, 2011.

[13] Del Dottore, Emanuela, et al. "Continuous growth in plant-inspired robots through 3D additive manufacturing." 2018 IEEE International Conference on Robotics and Automation (ICRA). IEEE, 2018.

[14] Mazzolai, Barbara, Lucia Beccai, and Virgilio Mattoli. "Plants as model in biomimetics and biorobotics: new perspectives." Frontiers in bioengineering and biotechnology 2 (2014): 2.

[15] Plooij, Michiel, et al. "Lock your robot: A review of locking devices in robotics." IEEE Robotics \& Automation Magazine 22.1 (2015): 106-117.

[16] Wright, Cornell, et al. "Design and architecture of the unified modular snake robot." 2012 IEEE International Conference on Robotics and Automation. IEEE, 2012. 